\title{
BMJ Quality Gout in primary care: Can we improve patient outcomes?
}

\author{
Jacqueline Callear, Georgina Blakey, Alexandra Callear, Linda Sloan
}

To cite: Callear J, Blakey G, Callear A, et al. Gout in primary care: Can we improve patient outcomes?. BMJ Quality Improvement Reports 2017;6:u210130.w4918. doi:10.1136/bmjquality. u210130.w4918

Received 27 April 2016 Revised 8 August 2016

\section{CrossMark}

Sloan Medical Practice, Sheffield

\footnotetext{
Correspondence to Jacqueline Callear jacquelinecallear@doctors. org.uk
}

\section{ABSTRACT}

In the United Kingdom, gout represents one of the most common inflammatory arthropathies predominantly managed in the primary care setting. Gout is a red flag indicator for cardiovascular disease and comorbidity. Despite this, there are no incentivised treatment protocols and suboptimal management in the primary care setting is common. A computer based retrospective search at a large inner city GP practice between January 2014-December 2014 inclusive, identified 115 patients with gout. Baseline measurements revealed multiple gout related consultations, poor medication compliance, high uric acid levels and deficiencies in uric acid monitoring.

A series of improvement cycles were conducted. A telephone questionnaire conducted in January 2015 , identified that patient education was suboptimal. The following improvement cycles aimed to educate patients, improve uric acid monitoring and support medication compliance. It was ultimately hoped that these measures would reduce gout flares and GP practice attendance. The improvement cycles contributed towards reduction in uric acid levels from 0.37 to 0.3 ( $p=0.14), 20 \%$ reduction in patients experiencing one or more gout flares and $77 \%$ reduction in GP related consultations between March 2015-March 2016 compared to baseline. The proportion of patients fully compliant with taking their urate lowering therapies improved from $63 \%$ to $91 \%$ $(p=0.0001)$.

A follow up series of PDSA cycles were performed between July-December 2016. The purpose of these cycles was to assess the sustainability of the improved medication compliance demonstrated by the improvement cycles. Three months following the completion of the improvement cycles, full medication compliance dropped from $91 \%$ to $70 \%$ ( $p=0.0001)$. The introduction of a paper calendar saw sustained and maintained improvement in medication compliance to $100 \%(p=0.0001)$ at the end of the study period. The improvement and PDSA cycles have demonstrated that simple interventions can be a sustainable way of improving disease control and patient outcomes.

\section{PROBLEM}

Gout remains a significant concern within general practice. The prevalence of gout in developed countries is predicted to remain stable or even increase in the near future. ${ }^{1}$ This is partially due to longevity, increased alcohol consumption, hypertension and obesity. $^{2}$ The National Institute for Health and Care Excellence (NICE) provides guidelines for general practitioners on the diagnosis and management of gout. ${ }^{3}$ Patient education and lifestyle advice, urate lowering therapy (ULT), assessment of cardiovascular disease risk and optimal uric acid levels are central to the recommendations. Patients should be given information about weight optimisation and dietary advice. Urate lowering therapy should be commenced after $\geq 2$ acute gout flares in the same year or following one flare in high risk individuals including those with $\geq 1$ tophi, nephrolithiasis, renal impairment and those on longstanding diuretic therapy. Serum urate should be $\leq 0.30 \mathrm{mmol} / 1^{4}$. NICE recommends that uric acid levels should be checked quarterly in the first year and then annually in patients taking allopurinol. ${ }^{3}$ The frequency of liver function testing in febuxostat is determined by the individual clinician. However, despite the presence of these guidelines and the availability of effective urate lowering therapies, audits have repeatedly revealed the suboptimal management of gout within primary care. $^{5-9}$

\section{BACKGROUND}

Gout is the most common inflammatory arthropathy in the United Kingdom (UK), affecting $1.5 \%$ of the population and contributing to approximately $4.9 \mathrm{UK}$ primary care consultations per 1000 adults per year. ${ }^{1}$ Gout is characterised by dysfunctional purine metabolism, which leads to hyperuricaemia and the consequent deposition of monosodium urate crystals in joints, connective tissues and the urinary tract. ${ }^{9}$ This urate deposition may lead to acute flares of painful gouty arthritis and the eventual development of chronic gouty tophi, which may irreversibly damage joints and hinder activities of daily living. Gout is becoming increasingly associated with cardiovascular risk factors and disease. ${ }^{10-16}$ 
Gout is predominantly managed in the primary care setting. However, gout is not currently included within the Quality Improvement Framework (QOF) targets used by UK General Practitioners (GPs). ${ }^{17}$ Currently the medical management of gout is often reactive rather than preventative. ${ }^{18}$

Seminog et al investigated the relationship between gout and cardiovascular disease in 201,033 English patients. ${ }^{13}$ Gout was associated with an $82 \%$ increased risk of myocardial infarction and a $71 \%$ increased risk of stroke. ${ }^{13}$ Although causal relationships between hyperuricaemic gout and cardiovascular disease are difficult to establish as several risk factors including male gender, increased age and obesity are common to both, this does suggest that gout is a marker for cardiovascular disease.

\section{BASELINE MEASUREMENT}

A computer based retrospective study was performed for a twelve month period between January 2014-December 2014. All patients with a coded diagnosis of gout and those prescribed hyperuricaemic agents with a clinical diagnosis of gout were included. The minimum age for inclusion was 18 years of age. Patients whose health was deteriorating and who were now on end of life health pathways were excluded. General demographic details were collated. Age, gender, ethnicity, body mass index, smoking status, alcohol consumption and 10-year cardiovascular disease scores were noted. Uric acid levels and the frequency of testing were evaluated. The number of documented gout flares and GP attendances per gout flare were collected. These were used a markers of disease control.

This search revealed 115 patients, 21 female, 94 male with a coded diagnosis of gout. Of the patients included in the cohort, the mean age was 65.6 years (range 33-91) and mean BMI was $29.2 \mathrm{~kg} / \mathrm{m} 2$ (range 20-47). $25.0 \%(\mathrm{n}=29)$ of patients had BMI $<=25 \mathrm{~kg} / \mathrm{m} 2,40 \%$ $(\mathrm{n}=46)$ of patients had BMI $>25 \mathrm{~kg} / \mathrm{m} 2<=30 \mathrm{~kg} / \mathrm{m} 2$ and $35 \%(\mathrm{n}=40)$ of patients had BMI $>30 \mathrm{~kg} / \mathrm{m} 2.86 \% \quad(\mathrm{n}=99)$ of patients were white British, 3.4\% $\quad(n=4)$ Afro-Caribbean, 2.6\% $(\mathrm{n}=3)$ white European, 2.6\% $(\mathrm{n}=3)$ white Irish, 2.6\% (n=3) Pakistani, 0.9\% ( $\mathrm{n}=1)$ Asian, $0.9 \%(\mathrm{n}=1)$ African and $0.9 \%(\mathrm{n}=1)$ Chinese.

The medical co-morbidity status of the patients with gout was examined. The co-morbidities that were analysed included chronic kidney disease, cardiovascular disease, hypertension, peripheral vascular disease and diabetes mellitus. $76 \%$ of patients, $(n=87)$ had one or more co-morbidity. $33 \%$ of patients $(n=38)$ had one co-morbidity, $25.6 \%$ of patients $(\mathrm{n}=30)$ had two co-morbidities, $14.8 \%(\mathrm{n}=17)$ had three co-morbidities and $3.5 \%(n=4)$ had four co-morbidities.

All patients in the cohort met the criteria for the prescription of urate lowering therapies. All patients were prescribed allopurinol. All allopurinol prescriptions were monthly, collected at the beginning of each month.
All patients had been taking allopurinol for longer than one year. Ninety one patients, $(79 \%)$ of all patients with a diagnosis of gout had a uric acid level measured in the last year. The mean uric acid level was $0.37 \mathrm{mmol} / \mathrm{L}$ (range 0.17-0.69).

Between January 2014 - December 2014, 22\% ( $n=25)$ of patients experienced a flare of gout. $29 \% \quad(n=6)$ of women experienced at least one flare of gout. 9.5\% $(\mathrm{n}=2)$ of women experienced one flare of gout leading to 2 attendances per flare. $9.5 \% \quad(n=2)$ of women experienced two flares of gout leading to 2 attendances per flare. $9.5 \%(n=2)$ of women experienced three flares of gout leading to 2.5 attendances per flare. These women experienced an average of 2 flares per annum. 20\% $(\mathrm{n}=19)$ of men experienced at least one flare of gout. $26 \%(n=5)$ of men experienced one flare of gout leading to 2.2 attendances per flare. $32 \%(n=8)$ of men experienced two flares of gout leading to 2.25 attendances per flare. $31.6 \%(n=6)$ of men experienced three flares of gout leading to 2.3 attendances per flare. These men experienced an average of 2.1 flares per annum.

During the baseline period, seventy-three patients $(63 \%)$, collected twelve repeat prescriptions, 22\% $(n=25)$ of patients collected six to 11 repeat prescriptions and $15 \%(\mathrm{n}=17)$ collected five repeat prescriptions or less.

Independent risk factors for gout flares were high urate levels $(\mathrm{p}=0.0001)$, poor medication compliance ( $p=0.004)$, younger age $(p=0.009)$, fewer co-morbidities $(\mathrm{p}=0.039)$ and lower cardiovascular disease risk (CVDr) scores $(p=0.038)$. Independent risk factors for gout related GP consultations were fewer co-morbidities $(p=0.0301)$, younger age $(p=0.013)$ and higher urate levels $(\mathrm{p}=0.00027)$.

\section{DESIGN}

The preliminary study results revealed several important findings. Patients with poor medication compliance and higher urate levels experienced more flares of gout. A decision was made to contact each patient in the study cohort by telephone. The aim of this consultation was to understand the reasons which were leading to poor disease control. Each patient's knowledge about gout and the role of urate lowering therapies was assessed. It was predicted that poor levels of understanding about urate lowering therapies may be contributing to poor disease control.

In the first week of January 2015, all patients were contacted by telephone and asked a series of questions:

1. What do you currently know about gout? Do you know any dietary and lifestyle recommendations that can help improve gout control?

2. Would you like to be more knowledgable about gout? Would a diet, lifestyle and advice sheet about gout help to improve your level of knowledge?

3. Are you aware of annual uric acid monitoring whilst taking allopurinol? 
4. Do you understand the role of allopurinol in gout management? Do you know that urate lowering therapies should be taken every day to prevent gout flares?

All answers to the above questions were recorded. $100 \%$ of respondents reported knowing very little about gout. The most commonly noted sources of information used by patients were internet based. Very few patients $(n=10)$, could name specific modifying lifestyle factors which could improve their disease control. Very few patients $(n=25)$, had received any written information from their general practitioner at the time of their diagnosis. Many patients, $(n=110)$, reported that their lack of knowledge of gout impaired their ability to manage their own condition. Although $79 \% \quad(n=91)$ of patients had their uric acid level measured within the last year, only $43 \% \quad(n=49)$ of patients were aware that this was necessary. Only $63 \%$ of patients $(n=73)$ knew that ULT were preventative and should be taken every day to reduce gout flares. $37 \%$ of patients $(n=42)$ thought that they did not need to take ULTs on a regular basis if they were not troubled by symptoms of gout. All patients were keen to receive a good quality, gout specific, diet, lifestyle and advice sheet. The telephone questionnaire identified patient education as an important area for improvement.

Three key areas were targeted by a series of improvement cycles. Firstly patient education needed to be improved. Secondly, the frequency of uric acid measurement for patients taking ULTs should reflect NICE guidance. ${ }^{3}$ Thirdly through patient education and lifestyle measures it was hoped there would be decrease in gout flares and GP visits. A series of PDSA cycles were commenced three months following the end of the improvement cycles. The purpose of the PDSA cycles was to evaluate current medication compliance and thereby evaluate how sustainable the results of the improvements cycles had been. Methods to sustain medication compliance were trialled and implemented.

\section{STRATEGY}

The aim of improvement cycle one was patient education. The baseline telephone consultations revealed that the patients with gout wanted more information about gout and how to reduce gout flares through medication, diet and lifestyle measures.

One week following the telephone consultation, a diet, lifestyle and advice sheet was sent to all the patients in the cohort. This included a brief overview about gout as a disease, to improve patient knowledge. There was also a section about relevant medications - those taken in an acute flare and those used as preventative medications. The final section discussed diet and lifestyle changes to minimise gout flares.

This was followed up by a telephone consultation at the end of January 2015, giving a minimum two week interval since the advice sheets had been received. All patients had received and read the advice sheet. Five follow up questions were asked:

1. Did you find the diet, lifestyle and advice sheet useful?

2. Has your medication compliance improved?

3. Will you comply with annual uric acid blood testing?

4. Do you have any further comments or questions? $100 \%$ of patients found the diet, lifestyle and advice sheet useful. All patients expressed that the sheet was user friendly, contained all the key information and was easily understandable. All patients reported better understanding of the role of ULTs. All patients agreed to attend for an annual blood test. Several patients said that they hoped they could reduce gout flares to minimise disruption to work.

The aim of the second improvement cycle was to improve compliance with annual uric acid blood testing. A secondary aim was to complete 10 year cardiovascular risk disease scores for all patients with gout. On the first week of February 2015 a meeting was held with the phlebotomy staff at the practice. A new gout order set was devised to include FBC, U\&E, cholesterol, urate, random blood sugar and HbAlc. A blood pressure measurement was incorporated into the phlebotomy appointment to complete the 10year CVD risk factor score if required. Letters were sent out to all patients who had not had a uric acid level taken within the last 12 months and those requiring blood pressure measurement. All patients were offered blood test and blood pressure appointments in the following three weeks. At the end of February, the proportion of patients that had a uric acid level and cardiovascular risk score calculated was analysed.

The aim of the third improvement cycle was to further improve uric acid measurement. A secondary aim was to improve medication compliance. By the end of improvement cycle 2 , only $83 \%$ of patients had a documented uric acid level within the previous 12 months. The remaining 20 patients were contacted by telephone. Seventeen of these patients $(85 \%)$ reported difficulty in attending phlebotomy appointments during the day due to work commitments. After discussion with the phlebotomy team, a number of early appointments were made to overcome this barrier. These appointments were made available in March 2015.

The diet, lifestyle and gout advice sheet was read by all patients. The information leaflet improved knowledge of annual uric acid testing which $100 \%$ of patients said they would attend if invited. At baseline, $79 \%$ of patients had a uric acid level measurement in the last year. $83 \%$ of patients had a uric acid measurement in the last year following improvement cycle 2 .

Following the three improvement cycles, a re-assessment of gout flares, number of GP attendances and recorded uric acid blood tests was performed. During April 2015-September 2015, 13\% ( $n=15)$ of all patients experienced a single flare of gout. No patients experienced multiple flares of gout. $10 \% \quad(n=2)$ of 
women experienced a flare of gout leading to 1.5 attendances per flare. This is compared to $14 \%(n=13)$ of men who attended 1.3 times per flare. Overall the reduction in the number of men and women experiencing gout flares was reduced by $66 \%$ and $32 \%$ respectively. The number of attendances per flare reduced for men and women by $46 \%$ and $29 \%$ respectively.

In the final week of September 2015, a practice meeting was held to discuss the early study results. Many members of the multidisciplinary team were in attendance: general practitioners, trainee registrars, medical students, nurses, health care assistants, the practice pharmacist and the practice manager. Those in attendance were interested to learn of the improvement in disease monitoring and control. However, there was recognition that this improvement had been reasonably resource intensive and there were questions about sustainability. It was decided that a further 6 month period of evaluation of gout flares, GP practice attendance and compliance with uric acid measurement was required.

Between October 2015-March 2016, 4.7\% $(n=1)$ of women experienced a flare of gout, leading to 1 attendance per flare. $4.2 \% \quad(n=4)$ of men experienced a flare of gout, leading to 1.5 attendances per flare. When the baseline measurement year January 2014-December 2014 was compared to the intervention year March 2015-March 2016, there was a 20\% reduction $(n=5)$ in the number of patients experiencing at least one flare of gout. However, interestingly there was only one patient who had two separate flares of gout, compared with 18 patients at baseline experiencing one or more gout flare. This represents a reduction of $94 \%$ in multiple gout flares per annum. The trend for gout flares is demonstrated in Table 1. As gout flares decreased, so did GP attendance. The trend for GP related consultations for gout are demonstrated in Table 2. At baseline, January 2014-December 2014 there were 115 GP appointments for gout, compared to just 27 appointments between March 2015-March 2016 during the intervention period. This represents a reduction in GP related consultations by $77 \%$. Average uric acid levels dropped from 0.37 at baseline, to 0.34 at the end of improvement cycle 2 and 0.30 at the end of improvement cycle 3. Although the mean uric acid level decreased to within the recommended level, this was not

\begin{tabular}{lll}
$\begin{array}{l}\text { Table } 1 \text { Number of patients experiencing more than one } \\
\text { flare of gout }\end{array}$ & $\begin{array}{l}\text { No of patients } \\
\text { experiencing 1+ } \\
\text { flares of gout }\end{array}$ & Percentage \\
\hline Time period & 25 & 22 \\
\hline $\begin{array}{l}\text { Baseline Jan } \\
\text { 14-Dec 14 }\end{array}$ & 15 & 13 \\
$\begin{array}{l}\text { April-September } \\
\text { 15 }\end{array}$ & 15 & 4 \\
Oct 15 - March 16 & 5 & \\
\hline
\end{tabular}

statistically significant. The trend for of uric acid measurement is detailed in Table 3 .

During the baseline measurement period, $63 \%(n=73)$ patients collected their full quota of pharmacy prescriptions. During the final study period, October 2015-March 2016, 91\% ( $\mathrm{n}=105)$ of patients had collected $100 \%$ of their repeat ULT prescription, whilst the remaining $9 \%(\mathrm{n}=10)$ had collected over $83 \%$ of their prescriptions.

In the final week of March 2016, a multi-disciplinary practice meeting was held to discuss the study results. Those in attendance were interested to hear of the improvement in gout flares, GP related consultations, reduction in uric acid levels and improved medication compliance. However, there was some uncertainty of the sustainability of the improvements demonstrated. It was felt that medication compliance was a key determinant factor to disease control. Therefore it was decided that a three month evaluation of medication compliance following the improvement cycles should be performed. If this demonstrated some deterioration of medication compliance, it was thought that further measures would be needed.

Between April to June 2016, medication compliance was re-assessed. $70 \%$ of patients $(\mathrm{n}=85)$ collected $100 \%$ of prescriptions. The remaining $30 \%(\mathrm{n}=35)$ of patients had collected $66 \%$ of prescriptions. During the baseline measurement period, January 2014-December 2014, prior to the improvement cycles, $63 \%(\mathrm{n}=73)$ of patients collected $100 \%$ of prescriptions, $22 \% \quad(n=25)$ collected six to eleven repeat prescriptions and $15 \%(\mathrm{n}=17)$ collected five repeat prescriptions or less. Whilst there was improvement in medication compliance when compared with the baseline data, there had been some deterioration in medication compliance since the end of the improvement cycles. Two PDSA cycles were designed to evaluate the reasons underpinning poor medication compliance and to design a sustainable method to improve this.

PDSA cycle one: In the last week of June, the 35 patients who had collected 2 prescriptions between April-June 2016 were contacted by telephone and invited to a group meeting about gout compliance. Twenty-three patients $(66 \%)$, were in attendance. The patients in the group said that they had found the interventions during the improvement cycle period, January 2014-December 2014 helpful and now better understood the role of urate lowering therapies. They were all keen

Table 2 Number of GP consultations for gout

\begin{tabular}{ll}
\hline Time period & $\begin{array}{l}\text { No of GP appoitments } \\
\text { for gout }\end{array}$ \\
\hline Baseline Jan 14-Dec 14 & 115 \\
April-September 15 & 20 \\
Oct 15 - March 16 & 7 \\
\hline
\end{tabular}


Table 3 Uric acid levels

\begin{tabular}{ll} 
Time period & Uric acid level \\
\hline Baseline Jan 14-Dec 14 & 0.37 \\
Completion improvement cycle 2 & 0.34 \\
Completion improvement cycle 3 & 0.3 \\
\hline
\end{tabular}

to strive towards $100 \%$ medication compliance. All of the patients in attendance stated that it was merely that they forgot to collect their prescription, rather than any specific difficulty with taking the medication. All patients felt that a physical reminder to collect and take their medication would be very useful. It was proposed that a monthly paper calendar with tick boxes for each day of the month would be a simple yet useful intervention. The calendar was offered in two different sizes, A4 to be displayed on a notice board or fridge and A6 that could be folded into the cardboard medication box or placed into a wallet. The patients were told to place a tick in the box for each day if they had taken their tablet. There was also a reminder inbuilt into the calendar which was a reminder to collect their repeat prescription. Patients were advised to tick this box when they had collected their repeat prescription. The calendars were issued to the 22 patients that attended the meeting. The calendars issued were for a six month time period.

Between July to September, of the twenty three patients that were using the calendar as a reminder, $100 \%$ of patients collected all of their prescriptions. The 85 patients that had collected all of their prescriptions between April-June 2016, again collected their full quota of prescriptions during this study period. The remaining twelve patients collected two of their three prescriptions. The twenty three patients in the intervention group were contacted by phone. All of the patients were using the calendar and were finding it to be an effective reminder to collect and take their medication. As the improvement in medication compliance was so effective in the intervention group. It was decided to invite the twelve remaining patients without perfect medication

\begin{tabular}{lcc} 
Table 4 & Collection of full complement of prescriptions \\
\hline & $\begin{array}{l}\text { Collection of full } \\
\text { complement of } \\
\text { prescriptions } \\
\text { (number of patients) }\end{array}$ & Percentage \\
Time period & 73 & 63 \\
\hline $\begin{array}{l}\text { Baseline Jan } \\
\text { 14-Dec 14 }\end{array}$ & 105 & 91 \\
$\begin{array}{l}\text { Oct 15 - March 16 } \\
\text { April - June 16 }\end{array}$ & 85 & 74 \\
$\begin{array}{l}\text { Completion PDSA } \\
\text { cycle 1 }\end{array}$ & 103 & 90 \\
$\begin{array}{l}\text { Completion PDSA } \\
\text { cycle 2 }\end{array}$ & 115 & 100 \\
\hline
\end{tabular}

compliance to a short meeting to discuss this and issue them with a calendar.

PDSA cycle two: On the last week of September, a meeting was held with the remaining twelve non compliant patients. All twelve patients were in attendance. Each patient was issued a calendar for documentation of medication compliance.

Between October to December 2016 the twelve patients that received the calendar on the final week of September, collected $100 \%$ of their prescriptions. The twenty three patients from PDSA cycle 1 also collected $100 \%$ of their prescriptions. The 85 patients that had collected their full prescription quota to date also collected $100 \%$ of their prescriptions.

The thirty five patients that had been in receipt of the calendar were contacted by telephone. The twenty three patients from PDSA cycle one were still finding the calendar a useful reminder for medication compliance and prescription collection. The twelve patients from PDSA cycle two were all finding the calendar useful in maximising their medication compliance. Following the second PDSA cycle all patients were issued with a twelve month calendar for 2017. By the completion of PDSA cycle 2, all patients were now collecting their full quota of prescriptions. The trend in patients collecting the full complement of prescriptions is detailed in Table 4 .

\section{RESULTS}

See supplementary file

\section{LESSONS AND LIMITATIONS}

The results measured in the improvement cycles were at times confounded by multiple interventions. Therefore the effect of individual interventions could not be measured. For example, in the second improvement cycle, a meeting with the phlebotomy team led to the development of a new gout order set. Invites were then sent to patients to attend for their annual blood test. Each of these interventions contributed towards improved compliance with annual uric acid level monitoring.

This project highlights that there are important medical conditions which require strict monitoring in primary care which lie outside the Quality and Outcome Framework targets (QOF). Gout has been highlighted as a condition that disproportionately affects young, working age people with few other medical co-morbidities. Simple methods of patient education including disease, diet and lifestyle information leaflets and telephone consultations can improve medication and blood test compliance. By improving disease control, disease morbidity can be reduced potentially lowering absence from work.

This project emphasised the importance of multidisciplinary working. Through engagement of general practitioners, registrars, phlebotomists and administrative staff, extra blood test appointments were provided 
our of hours to improve compliance with annual uric acid blood test monitoring. The appointments also allowed cardiovascular disease risk scores to be calculated and the patient to be considered more holistically. Patients verbally reported that telephone consultation was a useful method of communication as it prevented the need to attend the GP surgery.

The PDSA cycles were designed to assess the sustainability of the improved medication compliance seen during the improvement cycles. The use of monthly calendars to record patient medication compliance and the collection of repeat prescriptions, resulted in $100 \%$ medication compliance in the whole cohort group. This simple intervention was very effective.

For this intervention to continue to be successful, each patient with a new diagnosis of gout must receive education about gout and diet and lifestyle modifications. If urate lowering therapies are to be prescribed, the importance of good medication compliance and the schedule for blood tests must be discussed with the patient.

It is not always easy to introduce new patient management pathways as they can initially result in more workload for the participating practitioners. However, the interventions thus far have reduced GP consultation rates showing that a preventative approach is a successful way of managing gout as a chronic disease.

\section{CONCLUSION}

All patients with gout in primary care should receive treatment in accordance with the NICE guidelines. ${ }^{3}$ Each patient should be knowledgable about their condition and understand the importance of medication compliance, diet and lifestyle modifications. Early preventative measures may reduce gout flares, GP attendance and the risk of cardiovascular disease. This GP practice is currently discussing the development of an annual gout clinic. This would give general practitioners and patients opportunity to review their disease management over the previous year, medication compliance, biochemical monitoring and address any outstanding questions. The lead GP for this project is continuing this project with the support from the GP partners, phlebotomists and administrative staff and hopes the results will be sustainable.

Declaration of interests Nothing to declare.
Ethical approval This service improvement project was approved by the GP practice. No further ethical approval was sought.

Open Access This is an open-access article distributed under the terms of the Creative Commons Attribution Non-commercial License, which permits use, distribution, and reproduction in any medium, provided the original work is properly cited, the use is non commercial and is otherwise in compliance with the license. See:

- http://creativecommons.org/licenses/by-nc/2.0/

- http://creativecommons.org/licenses/by-nc/2.0/legalcode

1. Elliot AJ, Cross KW, Fleming DM. Seasonality and trends in the incidence and prevalence of gout in England and Wales 1994-2007. Ann Rheum Dis. 2009;68(11):1728-33.

2. Roddy $E$, Zhang W, Doherty $M$. The changing epidemiology of gout. Nat Clin Pract Rheumatol. 2007;3:443-9.

3. NICE. Gout. Clinical Knowledge Summaries. [Available from: http:// cks.nice.org.uk/gout\#!scenario:1]

4. Zhang W, Doherty M, Bardin T, Pascual E, Barskova V, Conaghan P . EULAR evidence based recommendations for gout. Part II: Management. Report of a task force of the EULAR Standing Committee for International Clinical Studies Including Therapeutics (ESCISIT). Ann Rheum Dis. 2006;65:1312-24.

5. Cottrell E, Crabtree V, Edwards JJ, Roddy E. Improvement in the management of gout is vital and overdue: an audit from a UK primary care medical practice. BMC Fam Pract. 2013;14:170.

6. Neogi T, Hunter DJ, Chaisson CE, Allensworth-Davies D, Zhang Y. Frequency and predictors of inappropriate management of recurrent gout attacks in a longitudinal study. J Rheumatol. 2006;33:104-9.

7. Pal B, Foxall M, Dysart T, Carey F, Whittaker M. How is gout managed in primary care? A review of current practice and proposed guidelines. Clin Rheumatol. 2000;19:21-5.

8. Wall GC, Koenigsfeld CF, Hegge KA, Bottenberg MM. Adherence to treatment guidelines in two primary care populations with gout. Rheumatol Int. 2010;30:749-53.

9. Doherty M. New insights into the epidemiology of gout. Rheumatology (Oxford). 2009;48 Suppl 2:ii2-ii8.

10. Abbott RD, Brand FN, Kannel WB, Castelli WP. Gout and coronary heart disease: the Framingham Study. J Clin Epidemiol. 1988:41:237-42.

11. Janssens $H J$, van de Lisdonk $E H$, Bor $H$, van den Hoogen $H J$, Janssen M. Gout, just a nasty event or a cardiovascular signal? A study from primary care. Fam Pract. 2003;20:413-6.

12. Mikuls TR, Farrar JT, Bilker WB, Fernandes S, Schumacher HR, Saag KG. Gout epidemiology: results from the UK General Practice Research Database, 1990-1999. Ann Rheum Dis. 2005;64:267-72.

13. Seminog OO, Goldacre MJ. Gout as a risk factor for myocardial infarction and stroke in England: evidence from record linkage studies. Rheumatology (Oxford). 2013:52:2251-9.

14. Krishnan E, Baker JF, Furst DE, Schumacher HR. Gout and the risk of acute myocardial infarction. Arthritis Rheum. 2006;54:2688-96.

15. Choi HK, Curhan G. Independent impact of gout on mortality and risk for coronary heart disease. Circulation. 2007;116:894-900.

16. Kuo CF, Yu KH, See LC, Chou IJ, Ko YS, Chang HC. Risk of myocardial infarction among patients with gout: a nationwide population-based study. Rheumatology (Oxford). 2013;52:111-7.

17. Employers N. 2014/15 General Medical Services (GMS) Contract Quality and Outcomes Framework (QOF), NHS England Gateway reference: 012642014 [Available from: http://www.nhsemployers.org/ /media/Employers/Documents/Primary care contracts/QOF/ 2014-15/14-15 General Medical Services contract - Quality and Outcomes Framework.pdf.]

18. L W. Why is gout so difficult to treat? $2012 \mathrm{http} / / / \mathrm{www}$.gponline.com/ guidelines-why-gout-so-difficult-treat/article/1159461. 